\title{
JUEGO PROBLEMA Y SINTOMATOLOGÍA DEPRESIVA EN ADOLESCENTES
}

\author{
ELISARDO BECOÑA, FERNANDO L. VÁZQUEZ y M. CARMEN MÍGUEZ \\ Universidad de Santiago de Compostela
}

(Aceptado en febrero de 2001)

\begin{abstract}
En adultos se ha encontrado una clara relación entre juego patológico y depresión. Sin embargo, en adolescentes esta relación no se ha estudiado. En el presente estudio se analiza esta relación en una muestra representativa de adolescentes escolarizados de ensenanza secundaria de Galicia $(N=2.790)$. Se evaluó el juego problema y juego de riesgo mediante el SOGS-RA de Winters et al. (1993) y la sintomatología depresiva con el Inventario de Depresión Infantil (CDI) de Kovacs (1992). Se encontró un incremento en la sintomatología depresiva conforme aumenta la implicación en el juego, con puntuaciones en el CDI de 12,71 para los no jugadores, 14,63 para los jugadores en riesgo y 19.66 para los jugadores problema. También aparecen diferencias por edad. Los que tienen mayores problemas de juego tienen mayores puntuaciones en sintomatología depresiva cuando se consideran las variables de mayor implicación de los padres en el juego, fracaso en los estudios y peores relaciones con sus padres. El conjunto de resultados muestra una relación consistente entre mayor implicación en el juego y mayor sintomatología depresiva.
\end{abstract}

Palabras clave: Juego problema, depresión, sintomatología depresiva, adolescentes.

\section{Problem gambling and depressive symptomatology in adolescents}

A clear relationship between gambling and depression has been found among adults. However, this relationship has not been studied among adolescents. The present study analyses this relationship in a representative sample of secondary school adolescents in Galicia ( $\mathrm{N}=2.790)$. Problem gambling and at- risk gambling were evaluated using the South Oaks Gambling Screen-Revised Adolescents (SOGS-RA) by Winters et al. (1993) and depressive symptomatology using the Child Depression Inventory, CDI, by Kovacs (1992). The results showed an increase in depressive symptomatology as involvement in gambling increases, with a CDI score of 12.71 for non-gamblers, 14.63 for at-risk gamblers and 19.66 for problem gamblers, with significant differences in all the groups. Differences were also found with respect to age. Those having greater gambling problems also show greater scores in depressive symptomatology when variables such as greater involvement of parents in gambling, academic failure, and worse relationship with their parents were considered. The results as a whole show a consistent relationship between greater involvement in gambling and greater depressive symptomatology.

Key words: Problem gambling, depression, depressive symptomatology, adolescents.

\section{INTRODUCCIÓN}

\section{A finales de los años 70 se legalizaron los bingos y casinos, en 1981 las máquinas tragaperras y posteriormente se hizo lo}

Correspondencia: Elisardo Becona, Universidad de Santiago de Compostela, Facultad de Psicologia, Departamento de Psicología Clínica y Psicobiología, Campus Universitario Sur, 15706 Santiago de Compostela.

Agradecimientos: Esta investigación ha sido financiade mediante el proyecto XUGA 21105B98 de la Secretaria de Investigación e Desenvolvemento de la Xunta de Galicia. Tenemos que agradecer a todos los Centros mismo con los otros juegos de azar que hoy conocemos. Cada año se incrementa la cantidad gastada en los juegos de azar. En España en el año 1999 se gastó en juego la cantidad de 3.935.252 millones de

(Colegios e Institutos), consejos escolares y directores el que nos hayan facilitado el acceso para hacer el estudio, y a los estudiantes por contestar el cuestionario. También a las Direcciones Provinciales de Educación de A Coruña, Lugo, Ourense y Pontevedra, a la Inspección de cada una de ellas y a la Dirección General de Ordenación Educativa y de Formación Profesional de la Consellería de Educación de la Xunta de Galicia por su colaboración y las facilidades proporcionadas para que la realización de este estudio fuese posible. 
pesetas; esto es, casi 4 billones de pesetas (Comisión Nacional del Juego, 2000). Esto representa un gasto por persona y año, en adultos, de 98.745 Pts. En torno a un $40 \%$ del dinero gastado en juego corresponde a las máquinas tragaperras, que como sabemos son las que producen un mayor nivel de adicción, de problemas de juego y de demanda de tratamiento (Becoña, 1996; Fernández-Montalvo y Echeburúa, 1997). Esto explica el que en España tengamos entre un 1,4\% y un $1,9 \%$ de jugadores patológicos (Becoña, 1999). Becoña, Labrador, Echeburúa, Ochoa y Vallejo (1995) estimaron en un $1,5 \%$ el número de jugadores patológicos adultos.

También se han realizado distintos estudios en adolescentes y jóvenes, tanto en otros países como en España, en los que se han encontrado niveles altos de juego problema y de juego patológico. Así, los porcentajes han oscilado entre el $1,7 \%$ en el estudio de Ladoucer y Mireault (1988) en Canadá, el $8 \%$ en el de Lesieur et al. (1991) en el estado de Nueva York y el 9,6\% en el estudio de Zitlow (1992) con adolescentes indios norteamericanos en Minnesota. Estudios recientes encontraron porcentajes entre el $3 \%$ y el $10 \%$ de jugadores problema en Estados Unidos (Govoni, Rupcich y Frisch, 1996; Westphal et al., 2000; Winters et al., 1993); del 4,7\% en Canadá (Gupta y Deverensky, 1998); y, del 5.6\% (Fisher, 1999); y, el 6\% (Woody y Griffiths, 1998) en el Reino Unido. Los primeros datos disponibles en España (Becoña, 1997) van en esa línea, con porcentajes entre el $2,4 \%$ y el $2,9 \%$ (p.ej., Arbinaga, 2000). Señalar, con respecto a estos estudios llevados a cabo con adolescentes, especialmente los realizados en la población para estimar su prevalencia, que en los últimos años se prefiere utilizar la denominación de juego problema en lugar de la de juego patológico (Winters, 2000; Winters, Stinchfield y
Fulkerson, 1993; Sproston, Erens y Orford, 2000) por varias razones: 1) por no ser posible establecer con los instrumentos utilizados hasta ahora un diagnóstico equivalente a los criterios diagnósticos del DSM-IV (suele utilizarse el SOGS o instrumentos derivados del SOGS que no son totalmente equivalentes a los criterios del DSM-IV); 2) porque todavía desconocemos la razón por la que se encuentra una prevalencia tan alta de juego patológico o juego problema en los estudios hechos con adolescentes, que suele ser del doble al cuádruple de la encontrada en adultos (Gambino, 1997; Yafee y Brodsky, 1997); 3) porque probablemente estemos sobreestimando la prevalencia del juego patológico en adolescentes, especialmente cuando se quiere hacer coincidir una evaluación puntual en la población de adolescentes mediante cuestionarios, respecto a lo que sería un diagnóstico clínico real de juego patológico (Gupta y Deverensky, 1998); y, 4) porque probablemente los instrumentos de evaluación que se vienen utilizando no son los más adecuados para este tipo de población (Gerstein et al., 1999), a pesar del gran desarrollo de instrumentos y estudios realizados en estos años en adolescentes (Winters, 2000). En nuestro estudio utilizaremos la denominación de jugador problema.

Dada la alta prevalencia de juego patológico, hay un interés creciente en encontrar variables que expliquen su aparición, su mantenimiento y su abandono. También es necesario conocer qué variables se relacionan con estas tres fases, ya sea en el sentido de no favorecer la aparición de conductas problemáticas de juego o el de favorecerlas. Se parte del hecho de que el juego está presente en nuestra sociedad. Si el juego no estuviese legalizado o no existiese los problemas apenas existirían (Becoña, 1999). La realidad actual nos dice que hay que aprender a convivir con el juego. Hoy en día, la 
enorme recaudación de impuestos y los puestos de trabajo creados, impiden un control más efectivo del mismo, afectando ello a la prevalencia y a los problemas de juego patológico.

Una de las cuestiones a la que más atención se le ha prestado es a la relación entre el juego patológico o el juego problema y la depresión. A nivel clínico es evidente que hay una clara relación entre juego patológico y depresión. Toda una serie de estudios, como se puede ver en distintas revisiones (p.ej., Becoña, 1993a, 1993b; Crockford y el-Guebaly, 1998), han encontrado que entre el $25 \%$ y el $75 \%$ de los jugadores patológicos, ya sea en estudios de tratamiento o epidemiológicos, tienen asociado un cuadro depresivo. Esta relación se ha hallado en estudios clásicos y bien diseñados como el de McCormick, Russo, Ramirez y Taber (1984), así como en estudios más recientes. Por ejemplo, Berg y Kühlhorn (1994) en Suecia, encontraron que el $53 \%$ de los jugadores patológicos que estaban tratando tenían una depresión severa. En estudios que se han realizado en España también se ha encontrado esta relación. En el estudio de Becoña, Lorenzo y Fuentes (1996), con una muestra de la población general, el $21 \%$ de los jugadores patológicos tenían depresión. García, Díaz y Aranda (1993) encontraron que el $16 \%$ de su muestra de jugadores patológicos cumplían los criterios de depresión mayor y el $41 \%$ habían tenido episodios depresivos antes de acudir al tratamiento.

Una cuestión de gran interés en este tema es saber si el juego se produce antes que la depresión o, viceversa, si la depresión es antes que el juego. Esto tiene una gran relevancia, no sólo teórica sino empírica, y cara al tratamiento y a los posibles tipos de tratamiento. Dos teorías específicas sobre la relación entre juego y depresión destacan aquí: la teoría de la adicción de Jacobs $(1986,1987,1989)$, la cual ha tenido y tiene una gran rele- vancia en Estados Unidos, y la teoría de la reversión de Brown (1986, 1987a, 1987b, 1988), la cual ha tenido y tiene más relevancia en Europa. La teoría de Jacobs plantea que la adicción es un estado dependiente adquirido en el tiempo por una persona predispuesta en su intento por aliviar una situación de estrés crónico. Habría dos niveles de predisposición: la activación psicofisiológica y la naturaleza psicológica, fruto de sus experiencias previas. Los que tienen un estado de arousal crónicamente anormal, hipotensivo (deprimido) o hipertensivo (excitado) y los que tienden a responder a los sentimientos de inferioridad y rechazo mediante fantasías compensatorias, son los que están en mayor riesgo de tener una adicción al juego. La teoría de Brown, más compleja para explicar en unas pocas líneas, también utiliza como elemento básico el arousal partiendo de la teoría de la reversión de Apter (1982). Con dos sistemas metamotivacionales (télico y paratélico), dos niveles de activación y dos niveles de tono hedónico (placer y reforzamiento), se pueden tener hasta cuatro posibles tipos de jugadores. En este caso los estados de ansiedad y depresión pueden explicarse a lo largo del tiempo en función de los resultados del juego, del nivel de activación y del tono hedónico. El objetivo del jugador es buscar el nivel óptimo de activación o arousal. Una ampliación de este modelo ha sido propuesto por Dickerson y Adcock (1987).Una descripción más amplia en castellano de estas teorías puede verse en Becoña (1993a) y Labrador y Becoña (1994).

Más actualmente destaca el modelo del estado negativo de Hand (1998). Sostiene que mientras que el jugador social está caracterizado por el reforzamiento positivo, el jugador patológico está bajo el reforzamiento negativo $y$, en los casos más graves, que él incluye bajo la denominación de juego adictivo, por la con- 
ducta presuicida. Habría dos características básicas del jugador patológico. La primera, sería como una estrategia para escapar, especialmente de los estados negativos, como la depresión, la ansiedad, los sentimientos de culpa, la ambivalencia, que le causan dolor y están continuamente presentes. Jugando evita estos estados negativos. La segunda, la carencia de sentido que tiene para él su vida. En este caso, el juego vendría a ser como una especie de tiempo fuera que le ayudaría a evitar los estados mentales negativos, la depresión y la ansiedad. Si deja de jugar se encuentra mal, tiene una gran urgencia de volver a jugar, se siente mal al no hacerlo y, además, no dispone de estrategias alternativas para poder mantenerse sin jugar. Conforme va avanzando más en la carrera adictiva, pasa al juego adictivo, con sentimientos de desesperación y ganas de morir, pérdida de sentido de su vida y otros problemas. Finalmente, juega para autodestruirse. Ahora, en este estado, la depresión ya forma parte del círculo vicioso juegodepresión.

Junto a las teorías anteriores, distintos autores han intentado responder a la cuestión de si se produce primero el problema del juego y luego el de la depresión o viceversa (p.ej., McCormick et al., 1984), pero sin mucho éxito. Parece que se pueden dar tres posibilidades: en unos casos la depresión conduce al juego; en otros el juego lleva a la depresión, especialmente cuando se ha perdido todo, no es posible acceder a más créditos y no se ve futuro; $y$, por último, que el juego y la depresión compartan una diátesis común. Aún así, McCormick et al. (1984) han llegado a proponer la existencia de un subtipo de jugador patológico donde la depresión es recurrente y que ésta precede al episodio de juego patológico. Según ellos, utilizarían el alto arousal que les produce el juego para aliviar su depresión. Otro subtipo sería el de bajo arousal. Posteriormente Blaszczynski, McConaghy y Frankova (1990) sugirieron añadir un tercer subtipo, aquel en el que los jugadores patológicos se caracterizan tanto por la depresión como por el aburrimiento.

La prevalencia de vida del trastorno depresivo mayor a lo largo de la adolescencia es alta, del $15 \%$ al $20 \%$, un porcentaje similar a los datos que se obtienen con los adultos (Kessler et al., 1994), que sugiere que la depresión en adultos puede tener su origen en la adolescencia (Harrington, Rutter y Fombonne, 1996). Además, es en las etapas media y final de la adolescencia (15-18 años) cuando hay un incremento en las tasas de depresión, un mayor riesgo de comienzo de la depresión y un incremento significativo en las diferencias por sexo en cuanto a las tasas de depresión (Hankin et al., 1998), hecho que no ocurre en la infancia (Olmedo, del Barrio y Santed, 2000). Por ello, muchos autores consideran básico conocer los procesos del desarrollo para poder comprender la depresión en los adolescentes (Cicchetti y Toth, 1998), y las múltiples variables que se asocian a la misma o que pueden actuar como factores de riesgo, desde los socio-culturales, biológicos, familiares, psicológicos, cognitivos, emocionales, etc. No es, por tanto, de extrañar que la psicopatología del desarrollo haya tenido un gran desarrollo especialmente en el campo de la depresión. Aun así, lo cierto es que hoy, al igual que lo que ocurre con el juego patológico, la mayoría de los niños y adolescentes no son tratados de su problema de depresión, a pesar de que hay tratamientos eficaces para la misma (Lewinsohn y Clarke, 2000; Méndez, 1998; Méndez, Moreno, Sánchez-Meca, Olivares y Espada, 2000).

Respecto al juego, y a las teorías antes expuestas, es de gran relevancia la depresión y la sintomatología depresiva de los sujetos, no sólo en adultos sino también 
en niños y adolescentes. Kovacs, Freinberg y Crouse-Noval (1984) señalan que hay algunos niños y adolescentes que están clínicamente inclinados a padecer síntomas depresivos. Su mayor sensibilización, por los episodios de depresión, los hace cada vez más susceptibles a los eventos vitales negativos. El juego, unido a la depresión o a la sintomatología depresiva, puede servir como una estrategia de escape. Pero, al mismo tiempo, al perder dinero y encontrarse sin posibilidad de acceso a más dinero, el mismo juego se convertiría en un evento negativo, cada vez más crónico conforme consigue dinero, juega y pierde el dinero. Esto llevaría a buscar dinero, pidiéndolo prestando, robándolo, etc., a implicarse más en el juego, descuidar el ir a clase o dar excusas para no ir, centrarse cada vez más en el juego y, finalmente, tener graves problemas de juego.

Además, hoy nos encontramos con un hecho nuevo, producto de la legalización del juego, ya que previamente no había jugadores patológicos o era una cuestión anecdótica. Algunos de los primeros jugadores excesivos, que empezaron a jugar a principios de los años 80 cuando tenían en torno a 18 años de edad, se convirtieron en jugadores patológicos en los siguientes años. Hoy son ya personas adultas de 40 años, muchos de ellos con hijos adolescentes. Además, los que comenzaron a jugar en los años siguientes tienen hoy hijos de pocos años o hijos también adolescentes. Dado que el juego de los padres influye en el de los hijos, una parte de éstos también serán jugadores patológicos, constituyendo la segunda generación de jugadores patológicos (ver Becoña, 1996). Este fenómeno da lugar a que los niños y adolescentes que juegan actualmente ya están en una parte importante de los casos influidos por el juego excesivo o el juego patológico de sus padres, además de existir una clara asociación entre mayor número de con- ductas adictivas de los padres y de juego patológico (Ochoa, Labrador, Echeburúa, Becoña y Vallejo, 1994). En ambos casos, la depresión también está presente en un porcentaje de casos, que puede ser de un $25 \%$ a un $50 \%$ de ellos (Berg y Kühlhorn, 1994).

Por tanto, a pesar de la gran cantidad de información que existe sobre la relación entre juego patológico y depresión, su relevancia clínica, y la necesidad de conocer la comorbilidad entre ambas, así como si existe una relación causal entre una y otra, especialmente en lo que atañe al campo del tratamiento, no se ha hecho hasta el momento ningún estudio en adolescentes para conocer si existe relación entre ambas. El objetivo del presente estudio es analizar la relación entre juego problema y depresión en una muestra representativa de adolescentes escolarizados.

\section{MÉTODO}

\section{Participantes}

La muestra incluyó 2.790 estudiantes de Educación Secundaria de Galicia que estaban cursando el 21 ciclo de la ESO, BUP, COU y FP-2, en Institutos (con o sin formación profesional) y en Colegios privados (con o sin formación profesional). Los que tenían más de 21 años ( $n=70$ ) no fueron incluidos en la muestra por su gran variabilidad de edades, como ocurrió especialmente en los que estaban cursando FP-2. El 45,6\% ( $n=1.272)$ eran varones y el $54,5 \%(n=1.518)$ mujeres. Los sujetos tenían edades comprendidas entre los 14 y los 21 años. La edad media de la muestra fue de 16,4 años (DT = 1,73).

La muestra se obtuvo seleccionando aleatoriamente los centros educativos de Galicia, considerando a todos, fuesen públicos o privados. La selección de los 
centros se hizo al azar mediante una tabla de números aleatorios, estratificándolos por provincia y tamaño del municipio (más de 50.001 habitantes, de 20.001 a 50.000 habitantes, de 10.001 a 20.000 habitantes, de 5.001 a 10.000 y menos de 5.000 habitantes). La muestra fue obtenida en 26 centros de distintas ciudades y pueblos de Galicia (10 en la provincia de A Coruña, 4 en la provincia de Lugo, 4 en la provincia de Ourense, y 8 en la provincia de Pontevedra). La selección de las clases en cada centro se hizo de modo aleatorio cuando en el centro había más de una por curso.

\section{Instrumentos de evaluación}

Se utilizó el cuestionario SOGS-RA de Winters, Stinchfield y Fulkerson (1993) para evaluar el juego problema y el juego de riesgo con la versión castellana de Becoña (1997). Este cuestionario consta de 12 items puntuables con dos alternativas de respuesta cada uno (SÍ/NO), derivado del SOGS de Lesieur y Blume (1987, 1993). Las puntuaciones del SOGS-RA nos proporcionan tres categorías: no jugador o sin problemas de juego, jugador en riesgo y jugador problema. Por no jugador o sin problemas de juego se entiende aquel que no contesta afirmativamente a ningún ítem o como máximo contesta a uno de ellos; jugador en riesgo se considera a aquel que contesta a 2 ó 3 de los items afirmativamente; y, jugador problema, sería aquel que contesta a 4 o más de ellos afirmativamente. Los que son jugadores problema tienen importantes problemas con el juego que les puede estar repercutiendo negativamente en su funcionamiento cotidiano, sea a nivel de estudios, relaciones con sus amigos, padres, etc., cumpliendo los criterios para la dependencia del juego tal como la conocemos en adultos. Los jugadores en riesgo estarían en un estado no tan grave, pero el juego les puede causar en el futuro o ya les puede estar causando problemas debidos al juego.

Junto al SOGS-RA se evaluaron en cada sujeto sus características demográficas, si juega alguno de los padres, si piensa que alguno de los padres juega demasiado, fracaso en los estudios y tipo de relaciones con sus padres (ver Tabla 1).

Para la evaluación de la depresión se utilizó el Inventario de la depresión infantil (Children Depression Inventory; CDI) de Kovacs (1992) en la versión castellana de del Barrio (1996). Es el cuestionario de depresión infantil más utilizado, tanto en otros países como en España (del Barrio y Moreno, 1996). Fue elaborado por Kovacs en 1978 , previa a la conceptualización realizada por Kovacs y Beck (1977). Posteriormente fue reelaborado por Kovacs (1992), siendo la versión que más se utiliza actualmente. Tiene cinco subescalas, las de humor negativo, problemas interpersonales, ineficacia, anhedonia y autoestima negativa. Los 27 items del cuestionario cubren contenidos de aspectos cognitivos, afectivos, fisiológicos y motivacionales de la depresión. Cada ítem tiene tres alternativas de respuesta de las cuales el sujeto tiene que elegir una. La puntuación va de 0 a 2 . El rango de puntuación oscila desde 0 a 54 . El punto de corte para población normal se ha estimado en 19 (Chartier y Larsen, 1994; del Barrio y Moreno, 1996; Olmedo, del Barrio y Santed, 2000), que es el que hemos utilizado en nuestro estudio, aunque en población clínica se puede bajar hasta 12 para evitar falsos negativos (Kovacs, 1992). Algunos autores españoles han apuntado la posibilidad de bajar el punto de corte a 17 (Polaino y García Villamisar, 1993). La fiabilidad es alta, de 0.92 en el estudio de Polaino y García-Villamisar (1993). 


\section{Procedimiento}

Previo a la realización del estudio, se solicitó permiso para la aplicación de los cuestionarios en los Centros a la Dirección General de Ordenación Educativa y Formación Profesional de la Consellería de Educación, a las delegaciones de la Consellería de Educación de cada provincia, a los directores de los centros y a sus respectivos consejos escolares. Se les indicó claramente que el cuestionario era anónimo y su cumplimentación voluntaria Una vez obtenida la autorización para realizar el estudio se concertaron las fechas y horas en las que los alumnos cumplimentarían el cuestionario. Este se pasó en los meses de febrero y marzo de 1999 en los colegios seleccionados. Dos psicólogos clínicos, que fueron entrenados especialmente para este estudio, se encargaron de aplicar el cuestionario en los centros seleccionados. Se pedía al profesor que abandonara el aula mientras los alumnos cubrían los cuestionarios. Se hacía la presentación del cuestionario, insistiendo en su carácter anónimo, dando las instrucciones precisas y específicas para la cumplimentación del mismo, se aclaraban las posibles dudas y, por último, los alumnos lo cumplimentaban.

\section{RESULTADOS}

\section{Tipo de jugador y sintomatología} depresiva

El cuestionario SOGS-RA tiene una fiabilidad en esta muestra (mediante el coeficiente alpha) de 0,83 . Se han utilizado los puntos de corte sugeridos por Winters et al. (1993) para el SOGS-RA (0-1, sin problemas de juego; 2-3 jugador en riesgo; 4 o más jugador problema). Los resultados indican que un $86,1 \%(n=2.403)$ del total de la muestra no tienen proble- mas de juego, un $8,2 \%(n=230)$ son jugadores en riesgo y un $5,6 \%(n=157)$ son jugadores problema para el período durante los últimos 12 meses. Respecto a las puntuaciones en depresión, la media del grupo es de 13,26 (DT = 6,79). La fiabilidad del CDI (coeficiente alpha) en esta muestra es de 0,84. En la Tabla 1 se indican las puntuaciones del CDI en función de distintas variables. Se encuentran diferencias significativas por sexo (mayor puntuación en las mujeres), juega alguno de los padres, alguno de los padres juega demasiado, mayor fracaso en los estudios y peores relaciones con sus padres. En la Tabla 2, se muestran las diferencias por sexo en el CDI en función de la edad. Los resultados indican un incremento de la sintomatología depresiva con la edad desde los 14 hasta los 18 años y luego una ligera disminución o estancamiento. Por sexo, las diferencias significativas se hallan en las edades de 14, 15 y 17 años, aunque a lo largo de todas las edades hay una clara tendencia de las mujeres a puntuar más en sintomatología depresiva que los varones.

La relación entre una mayor implicación en el juego y una mayor sintomatología depresiva también es clara. Los que no tienen problemas de juego tienen una puntuación de 12,71 (DT $=6,31$ ), que se incrementa a $14,63(\mathrm{DT}=6,95)$ en los jugadores en riesgo y a 19,66 (DT $=$ 19,66 ) en los jugadores problema. La diferencia es estadísticamente significativa entre los tres grupos, $F(2,2787)=$ $87,37, p<0,001$, y a su vez es significativa entre cada uno de los grupos entre sí (prueba de Scheffe, $p<0,05$ ); en concreto, entre el grupo de los que no tienen problemas de juego y los jugadores en riesgo, entre los que no tienen problemas de juego y los jugadores problema y entre los jugadores en riesgo y jugadores problema.

$\mathrm{El}$ análisis de las distintas variables consideradas en el estudio, en función de 
Tabla 1. Puntuaciones en el CDI en función de diversas variables

\begin{tabular}{|c|c|c|c|}
\hline Variables & $M$ & $D T$ & $F$ \\
\hline Muestra & 13,26 & 6,79 & \\
\hline $\begin{array}{l}\text { Sexo } \\
\text { Varón } \\
\text { Mujer }\end{array}$ & $\begin{array}{l}12,58 \\
13,83\end{array}$ & $\begin{array}{l}6,94 \\
6,61\end{array}$ & $23,54^{\star \star \star}$ \\
\hline $\begin{array}{l}\text { Edad } \\
14-17 \\
18-21\end{array}$ & 13,14 & $\begin{array}{r}6,80 \\
13,63\end{array}$ & $\begin{array}{l}2,68 \text { n.s. } \\
6,75\end{array}$ \\
\hline $\begin{array}{l}\text { Estudios } \\
\text { Instituto } \\
\text { FP }\end{array}$ & 13,26 & $\begin{array}{r}6,85 \\
13,27\end{array}$ & $\begin{array}{l}0,00 \text { n.s. } \\
6,50\end{array}$ \\
\hline $\begin{array}{l}\text { Juega alguno d } \\
\text { Si } \\
\text { No }\end{array}$ & $\begin{array}{l}13,33 \\
13,00\end{array}$ & $\begin{array}{l}6,45 \\
6,91\end{array}$ & 4,16 * \\
\hline $\begin{array}{l}\text { Alguno de los } \\
\text { Sí } \\
\text { No }\end{array}$ & $\begin{array}{l}17,54 \\
13,06\end{array}$ & $\begin{array}{l}7,61 \\
6,67\end{array}$ & $18,57 * * *$ \\
\hline $\begin{array}{c}\text { Fracaso en los } \\
\text { Ninguno } \\
\text { Uno o más }\end{array}$ & $\begin{array}{l}12,25 \\
14,78\end{array}$ & $\begin{array}{l}6,22 \\
7,31\end{array}$ & $96,13^{\star \star \star *}$ \\
\hline $\begin{array}{c}\text { Relaciones con } \\
\text { Malas } \\
\text { Normales } \\
\text { Buenas }\end{array}$ & $\begin{array}{l}20,18 \\
15,64 \\
11,85\end{array}$ & $\begin{array}{l}9,18 \\
6,66 \\
6,01\end{array}$ & 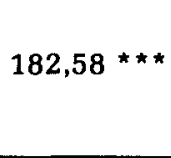 \\
\hline
\end{tabular}

Nota: "Se ha eliminado para el análisis la categoría de "lo ignoro". Los datos se presentan para las categorías de "si" y "no". * $p<0,05 ;{ }^{\star \star \star} p<0,001$

Tabla 2. Diferencias por sexo en el CDI en función de la edad

\begin{tabular}{lccccccc}
\hline \multirow{2}{*}{ Edad } & \multicolumn{3}{c}{ Muestra } & \multicolumn{3}{c}{ Hombre } & \multicolumn{2}{c}{ Mujer } & \\
\hline 14 años & Media & $D T$ & Media & $D T$ & Media & $D T$ & $t$ (sexo) \\
15 años & 11,61 & $\mathbf{6 , 6 3}$ & 10,74 & $\mathbf{5 , 7 1}$ & 12,41 & 7,29 & $2,49^{\star}$ \\
16 años & 12,68 & 6,72 & 11,93 & 6,98 & 13,36 & 6,42 & $2,477^{\star}$ \\
17 años & 13,64 & 6,59 & 13,30 & 6,75 & 13,94 & 6,44 & 1,21 n.s. \\
18 años & 14,04 & 7,03 & 12,96 & 7,08 & 14,93 & 6,87 & $3,34 \star \star$ \\
19 años & 14,51 & 7,14 & 14,06 & 8,13 & 14,87 & 6,22 & 1,00 n.s. \\
20 años & 12,76 & 5,93 & 11,85 & 5,56 & 13,48 & $\mathbf{6 , 1 3}$ & 1,87 n.s." \\
21 años & 12,96 & 6,32 & 12,88 & 7,62 & 13,01 & 5,32 & 0,10 n.s. \\
\hline
\end{tabular}

Nota: ${ }^{\star} p<0,05 ;{ }^{\star \star} p<0,01 ;{ }^{a} p=0,06$

los tipos de jugadores (ver Tabla 3), muestra consistentemente diferencias significativas en las mismas a lo largo de los grupos considerados, con mayores puntuaciones en depresión para aquellos que tienen una mayor implicación en el juego. Así, por sexo, hay un incremento en la sintomatología depresiva tanto en hombres como en mujeres según la mayor implicación en el juego. Las diferencias son significativas tanto para tipo de jugador, $F(2,2784)=74,72, p<0,001$, como sexo, $F(1,2784)=22,12, p<0,001$. Por edades, hay diferencias significativas en función del tipo de jugador, $F(2$, $2784)=61,24, p<0,001$, aunque no entre las edades consideradas (14-17 y 18-21 años). En estudios, hay diferencias significativas en el tipo de jugador, $F(2,2784)$ $=66,92, p<0,001$, aunque no en el tipo de centro (instituto o FP), pero sí aparece una diferencia significativa entre tipo de 
jugador $\mathrm{x}$ tipo de centro, $F(2,2784)=$ $3,36, p<0,05$. En la variable si juega alguno de tus padres, hay diferencias significativas en el tipo de jugador, $F(2$, $2583)=76,38, p<0,001$, no lo hay en esa variable y tampoco en la interacción tipo de jugador por dicha variable. En la variable si piensa que sus padres juegan demasiado, hay diferencias significativas por tipo de jugador, $F(2,2632)=13,46, p$ $<0,001$, en dicha variable, $F(1,2638)=$ $7,54, p<0,05$, pero no entre el tipo de jugador y la variable en cuestión.

En el fracaso en los estudios hay diferencias significativas en función del tipo de jugador, $F(2,2784)=77,22, p<0,001$, $\mathrm{y}$ en no tener ningún suspenso o uno o más, $F(1,2784)=33,82, p<0,001$. Finalmente, en las relaciones con sus padres, hay diferencias significativas en tipo de jugador, $F(2,2781)=67,84, p<0,001$, peores relaciones con sus padres, $F(2$, 2781) $=60,42, p<0,001$, e interacción de tipo de jugador y las relaciones con sus padres, $F(4,2781)=8,69, p<0,001$. (ver Tabla 4).

\section{Sospecha de depresión e implicación en el juego}

Otra importante relación a analizar es comprobar si aquellos de los que se sospecha que puedan tener depresión, tal como lo evalúa el CDI, siguiendo los estudios que se han hecho en otros países como en el nuestro, tiene relación con la mayor implicación con el juego.

Tabla 3. Medias y desviaciones típicas en sintomatología depresiva (CDI) en los distintos tipo de jugadores en distintas variables

\begin{tabular}{|c|c|c|c|c|c|c|}
\hline \multirow[b]{2}{*}{ Variables } & \multicolumn{2}{|c|}{ No jugador } & \multicolumn{2}{|c|}{$\begin{array}{l}\text { Jugador } \\
\text { en riesgo }\end{array}$} & \multicolumn{2}{|c|}{$\begin{array}{l}\text { Jugador } \\
\text { problema }\end{array}$} \\
\hline & $M$ & $D T$ & $M$ & $D T$ & $M$ & $D T$ \\
\hline Muestra & 12,71 & 6,31 & 14,63 & 6,95 & 19,66 & 9,53 \\
\hline $\begin{array}{l}\text { Sexo } \\
\text { Varón } \\
\text { Mujer }\end{array}$ & $\begin{array}{l}11,49 \\
13,53\end{array}$ & $\begin{array}{l}6,01 \\
6,38\end{array}$ & $\begin{array}{l}13,57 \\
17,55\end{array}$ & $\begin{array}{l}6,23 \\
7,99\end{array}$ & $\begin{array}{l}19,31 \\
21,48\end{array}$ & $\begin{array}{l}9,66 \\
8,73\end{array}$ \\
\hline $\begin{array}{l}\text { Edad } \\
\quad 14-17 \\
18-21\end{array}$ & $\begin{array}{l}12,54 \\
13,22\end{array}$ & $\begin{array}{l}6,35 \\
6,15\end{array}$ & $\begin{array}{l}14,92 \\
13,68\end{array}$ & $\begin{array}{l}7,28 \\
5,71\end{array}$ & $\begin{array}{l}19,26 \\
21,29\end{array}$ & $\begin{array}{r}8,63 \\
12,58\end{array}$ \\
\hline $\begin{array}{l}\text { Estudios } \\
\text { Instituto } \\
\text { FP }\end{array}$ & $\begin{array}{l}12,72 \\
12,64\end{array}$ & $\begin{array}{l}6,40 \\
5,89\end{array}$ & $\begin{array}{l}14,78 \\
13,64\end{array}$ & $\begin{array}{l}6,99 \\
6,67\end{array}$ & $\begin{array}{l}19,05 \\
22,34\end{array}$ & $\begin{array}{l}9,71 \\
8,30\end{array}$ \\
\hline $\begin{array}{l}\text { Juega alguno } d \\
\text { Sí } \\
\text { No }\end{array}$ & $\begin{array}{l}12,85 \\
12,48\end{array}$ & $\begin{array}{l}6,00 \\
6,54\end{array}$ & $\begin{array}{l}14,56 \\
14,05\end{array}$ & $\begin{array}{l}6,28 \\
6,71\end{array}$ & $\begin{array}{l}18,85 \\
20,05\end{array}$ & $\begin{array}{l}9,71 \\
9,01\end{array}$ \\
\hline $\begin{array}{l}\text { Alguno de los p } \\
\text { Si } \\
\text { No }\end{array}$ & $\begin{array}{l}16,11 \\
12,63\end{array}$ & $\begin{array}{l}5,55 \\
6,32\end{array}$ & $\begin{array}{l}18,25 \\
14,41\end{array}$ & $\begin{array}{l}7,26 \\
6,64\end{array}$ & $\begin{array}{l}20,23 \\
19,00\end{array}$ & $\begin{array}{r}10,67 \\
9,56\end{array}$ \\
\hline $\begin{array}{c}\text { Fracaso en los } \\
\text { Ninguno } \\
\text { Uno o más }\end{array}$ & $\begin{array}{l}11,89 \\
14,04\end{array}$ & $\begin{array}{l}6,04 \\
6,50\end{array}$ & $\begin{array}{l}13,53 \\
15,76\end{array}$ & $\begin{array}{l}5,74 \\
7,88\end{array}$ & $\begin{array}{l}17,67 \\
21,30\end{array}$ & $\begin{array}{r}7,63 \\
10,61\end{array}$ \\
\hline $\begin{array}{l}\text { Relaciones con } \\
\text { Malas } \\
\text { Normales } \\
\text { Buenas } \\
\end{array}$ & $\begin{array}{l}18,63 \\
15,34 \\
11,40 \\
\end{array}$ & $\begin{array}{l}7,39 \\
6,46 \\
5,65 \\
\end{array}$ & $\begin{array}{l}16,44 \\
16,00 \\
13,65 \\
\end{array}$ & $\begin{array}{l}7,06 \\
7,06 \\
6,75 \\
\end{array}$ & $\begin{array}{l}30,68 \\
19,06 \\
17,28 \\
\end{array}$ & $\begin{array}{r}11,36 \\
7,78 \\
7,91 \\
\end{array}$ \\
\hline
\end{tabular}

Nota: "Se ha eliminado para el análisis la categoría de "lo ignoro". Los datos se presentan para las categorías de "sí" y "no". * $p<0,05 ;{ }^{* \star} p<0,001$ 
Tabla 4. Resultados del análisis de varianza $(F)$ entre tipos de jugadores y las variables consideradas

\begin{tabular}{|c|c|c|c|}
\hline & Tipo de jugador & Variable & $\begin{array}{c}\text { Interacción } \\
\text { de jugador } \times \text { variable } \\
\end{array}$ \\
\hline Muestra & $87,37 \star \star \star$ & & \\
\hline Sexo & $74,72^{\star \star \star}$ & $22,12 \star \star \star$ & 1,85 n.s. \\
\hline Edad & 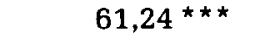 & 0,75 n.s. & 2,22 n.s. \\
\hline Estudios & $66,92 * \star \star \star$ & 1,20 n.s. & 3,36 * \\
\hline Juega alguno de los padres & $76,38^{\star \star \star}$ & 0,04 n.s. & 1,01 n.s. \\
\hline Algunos de los padres juega demasiado & 13,46 *** & 7,54 n.s. & $0,69 \mathrm{n} . \mathrm{s}$. \\
\hline Fracaso en los estudios & $77,22 * \star *$ & 33,82 *** & 0,93 n.s. \\
\hline Relaciones con los padres & $67,84 * \star *$ & $60,42 * \star \star$ & $8,69 * \star \star$ \\
\hline
\end{tabular}

Nota: ${ }^{\star} p<0,05 ;{ }^{\star \star \star} p<0,001$

Para hacer esto hemos utilizado el punto de corte recomendado para este tipo de estudios, de 19 o más para el CDI. Con ello tenemos dos grupos de personas, los que no se sospecha que tengan depresión, con una puntuación de 0 a 18 y los que se sospecha que tienen depresión con una puntuación de 19 o más. Partiendo de esta categorización, tal como podemos ver en la Tabla 5, volvemos a encontrar una relación aun más clara entre mayor sospecha de depresión y mayor implicación en el juego. Así, nos encontramos que mientras que en los no jugadores el porcentaje de personas con sospechas de depresión es de un $16,5 \%$, asciende a un $23,5 \%$ en los jugadores en riesgo, y pasa a ser casi la mitad de los jugadores problema, con un $47,8 \%$. La diferencia entre los tres grupos en tener o no depresión es estadísticamente significativa, $\chi^{2}(2, N=2.790)=98,01, p<$ 0,001 . Si analizamos esta misma relación independientemente en función del sexo (Tabla 6), para cada tipo de jugador, encontramos que hay diferencias significativas en los no jugadores, $\chi^{2}(1, \mathrm{~N}=$ $2.790)=30.17, p<0,001$, y en los jugadores en riesgo, $\chi^{2}(1, N=2.790)=16,94$, $p<0,001$, con un mayor porcentaje de sospecha de depresión en mujeres que en varones. En cambio en los jugadores problema no hay diferencias significativas por sexo. De modo semejante (Tabla 7) no aparecen diferencias significativas por edades en los distintos tipos de jugadores cuando consideramos a aquellos sospechosos o no de tener depresión.

\section{DISCUSIÓN}

Hoy sabemos que el juego patológico, como trastorno de salud mental en adultos, y el juego problema en adolescentes, constituye un grave problema. Este estudio confirma este dato en adolescentes. Se encontraron altos porcentajes de jugadores problema y jugadores en riesgo en una muestra representativa de

Tabla 5. Sospecha de depresión e implicación en el juego

\begin{tabular}{lrrrrr}
\hline & \multicolumn{5}{c}{$C D I$} \\
\cline { 2 - 5 } & $n$ & $0-18$ & $\%$ & $n$ & $19-54$ \\
\hline No jugador & 2007 & 83,5 & 396 & $\%$ \\
Jugador en riesgo & 176 & 76,5 & 54 & 23,5 \\
Jugador problema & 82 & 52,2 & 75 & 47,8 \\
\hline TOTAL & 2265 & & 525 & \\
\hline
\end{tabular}

Nota: $\chi^{2}(2)=98,01, p<0,001$ 
Tabla 6. Sospecha de depresión e implicación en el juego en función del sexo

\begin{tabular}{|c|c|c|c|c|c|}
\hline \multirow[b]{3}{*}{ Tipo de jugador } & \multicolumn{5}{|c|}{$C D I$} \\
\hline & \multicolumn{2}{|c|}{$0-18$} & \multicolumn{2}{|c|}{$19-54$} & \multirow[b]{2}{*}{$\chi^{2}$} \\
\hline & $n$ & $\%$ & $n$ & $\%$ & \\
\hline No jugador & & & & & $30,17 \star \star \star$ \\
\hline Varón & 860 & 88,6 & 111 & 11,4 & \\
\hline Mujer & 1147 & 80,1 & 285 & 19,9 & \\
\hline Jugador en riesgo & & & & & 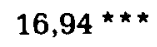 \\
\hline Varón & 141 & 83,4 & 28 & 16,6 & \\
\hline Mujer & 35 & 57,4 & 26 & 42,6 & \\
\hline Jugador problema & & & & & 0,81 n.s. \\
\hline Varón & 71 & 53,8 & 61 & 46,2 & \\
\hline Mujer & 11 & 44,0 & 14 & 56,0 & \\
\hline
\end{tabular}

Nota: ${ }^{\star \star *} p<0,001$.

Tabla 7. Sospecha de depresión e implicación en el juego en función de la edad

\begin{tabular}{|c|c|c|c|c|c|}
\hline \multirow[b]{3}{*}{ Tipo de jugador } & \multicolumn{5}{|c|}{$C D I$} \\
\hline & \multicolumn{2}{|c|}{$0-18$} & \multicolumn{2}{|c|}{$19-54$} & \multirow[b]{2}{*}{$\chi^{2}$} \\
\hline & $n$ & $\%$ & $n$ & $\%$ & \\
\hline No jugador & & & & & 1,16 , n.s. \\
\hline $14-17$ & 1521 & 84,0 & 290 & 16,0 & \\
\hline $18-21$ & 486 & 82,1 & 106 & 17,9 & \\
\hline Jugador en riesgo & & & & & 0,06 , n.s. \\
\hline $14-17$ & 134 & 76,1 & 42 & 23,9 & \\
\hline 18-21 & 42 & 77,8 & 12 & 22,2 & \\
\hline Jugador problema & & & & & 0,22 n.s. \\
\hline $14-17$ & 67 & 53,2 & 59 & 46,8 & \\
\hline $18-21$ & 15 & 48 & 16 & 51,6 & \\
\hline
\end{tabular}

estudiantes de enseñanza secundaria de Galicia. También se halló una clara relación entre juego y sintomatología depresiva, evaluada por el Inventario de depresión infantil (CDI), siendo ésta mayor a medida que la implicación en el juego también era mayor. Concretamente, el incremento en sintomatología depresiva va desde 12,71 en los no jugadores hasta 14,73 en los jugadores en riesgo y a 19.66 en los jugadores problemas. Es más, utilizando como punto de corte 19 para diferenciar entre los que se sospecha que pueden tener depresión o no, tal como se recomienda en este tipo de estudios (p.ej., Chartier y Larsen, 1994; Olmedo et al., 2000), hubo un porcentaje significativamente mayor de personas sospechosas de padecer depresión en los que tenían una mayor implicación en el juego. En el caso de sospecha o no de depresión, en el grupo de no jugadores hay un $16,5 \%$ de sospechosos de depresión, que aumenta a un $23,5 \%$ en los jugadores en riesgo y a un $47,8 \%$ en los jugadores problema, o lo que es lo mismo, casi el doble, de igual modo que se encuentra en adultos (p.ej., Berg y Kühlhorn, 1994).

Este estudio muestra como a partir de la edad de 14 o más años empieza a incrementarse la sintomatología depresiva y la depresión en las personas, lo cual coincide con lo encontrado en otros estudios. Así, por ejemplo, en el estudio de Carrasco, del Barrio y Rodríguez (2000), en una muestra representativa de la ciudad de Sevilla, con niños de 12 a 16 
años, se obtuvo una media en sintomatología depresiva utilizando el CDI de 11,95 (DT $=5,67$ ), siendo para los varones de $11,53(D T=5,55)$ y para las mujeres de 12,39 (DT $=5,77$ ). Por edades, se encontró un incremento consistente en sintomatología depresiva conforme aumentaba la edad: 12 años $(10,34 ; \mathrm{DT}=$ $5,46), 13$ años (11,53; DT = 6,19), 14 años $(12,44 ; \mathrm{DT}=5,46), 15$ años $(12,45 ; \mathrm{DT}=$ $5,63), 16$ años $(13,39$; DT $=4,97)$. Estos resultados son semejantes a los de nuestro estudio, en el que a los 14 años la media en el CDI fue de 11,61 , a los 16 subió a 13,64 , llegando a su punto más álgido a los 18 años con una puntuación de 14,51. Dado que este período coincide con la pubertad, con la experimentación con distintas sustancias y también con el comienzo en la participación en distintos juegos de azar, el juego puede tener funciones semejantes a las que se han encontrado en los adultos (p.ej., estrategia de escape) o bien pueden servir como un elemento activador del humor como han propuesto varias teorías y estudios que hemos revisado. Téngase en cuenta que uno de los criterios diagnósticos del DSM-IV para el juego patológico es el de Aescape@ (American Psychiatric Association, 1994), el cual recoge el hecho de que algunas personas pueden utilizar el juego como una forma de escapar de sus experiencias vitales negativas y como un modo de elevar su humor. De ahí la relevancia de este hecho, que en parte se ve confirmado en nuestro estudio porque, por edades, en los jugadores problema, la puntuación de las personas de 14 a 17 años es de 19,26, mientras que se incrementa a 21,29 en el grupo de edad de 18 a 21 años, aunque no se hallan encontrado diferencias significativas entre ambos grupos de edad.

Otras relaciones que hemos encontrado en este estudio atañen al propio estado del sujeto, con una mayor o menor sintomatología depresiva, que se expresa cuando esta es mayor y la persona tiene problemas de juego, un mayor nivel de fracaso en los estudios, peores relaciones con sus padres, relación de sus problemas con una percepción de mayor juego por parte de sus padres, etc. De ahí la relevancia de detectar a estar personas, con problemas de depresión, o con problemas de juego problema o juego patológico para hacer una adecuada intervención con ellos para que estas conductas no le acarreen problemas inmediatos (p.ej., fracaso en los estudios), a medio (p.ej., suspender el curso académico) o a largo plazo (p.ej., depresión clínica, juego patológico, mayores problemas psicopatológicos, menores habilidades antes la vida, etc.).

Cara a el futuro es de gran relevancia poder conocer algún tipo de mecanismo causal entre la depresión y el juego patológico. Hoy no conocemos si la depresión es previa al juego patológico, o si el juego patológico es previo a la depresión o si ambos tienen una diátesis común. Distintos modelos teóricos han intentado contestar a esta pregunta, aunque sin mucho éxito. También es necesario clarificar si estamos hablando de tipos de sujetos, en donde en unos casos la depresión puede llevar más fácilmente al juego problema o patológico, por la gran disponibilidad de juegos de azar, o si el perder en los juegos de azar la persona tiene una mayor probabilidad de caer en un cuadro depresivo, o si hay algún otro mecanismo que hoy no conocemos que nos pueda ayudar a explicar más adecuadamente esta relación. Aun así, lo que demuestra este estudio es que hay una relación evidente entre el juego problema en adolescentes y la depresión. $\mathrm{Y}$, que por la evidencia que tenemos en adultos, esta relación hay que comenzar a estudiarla a edades tempranas, como en las edades que cubre este estudio o incluso a edades previas. Conocer dichos mecanismos sería de gran ayuda tanto para el diseño de programas preventivos para el 
juego patológico (Secades y Villa, 1998) como para un mejor diseño de los programas de tratamientos de la depresión para adolescentes, ya que en adultos la evaluación y tratamiento de la depresión es un elemento más del tratamiento. Dado que sabemos que la depresión va en aumento en las sociedades avanzadas (Kessler et al., 1994; Lewinsohn y Clarke, 2000), cobra gran relevancia saber si este incremento se debe a factores del individuo o a factores ambientales, entre los que la accesibilidad a los juegos de azar puede tener su importancia. Nótese que en España, la amplia variedad de juegos, el dinero que se gasta en jugar y el tener una amplia accesibilidad al juego, facilita el continuo incremento de la conducta de juego en adolescentes cuando paradójicamente no está permitido jugar legalmente antes de los 18 años. $Y$ sabemos, como indica por ejemplo Winters (2000), que en las edades jóvenes hay un porcentaje muy elevado de jugadores problema, teniendo estas personas un alto riesgo de desarrollar juego patológico en los años sucesivos, aunque no necesariamente lo van a desarrollar todos.

\section{REFERENCIAS BIBLIOGRÁFICAS}

American Psychiatric Association (1994). Diagnostic and statistical manual of mental disorders (DSM-IV) ( $4^{\text {th }}$ ed.). Washington, DC: Author.

Apter, M.J. (1982). The experience of motivation: The theory of psychological reversals. London: Academic Press.

Arbinaga, F. (2000). Consumo de drogas y juego patológico en Punta Umbría (Huelva). Revista Española de Drogodependencias, 25, 301-320.

Becoña, E. (1993a). El juego compulsivo en la comunidad autónoma gallega. Santiago de Compostela: Consellería de Sanidade da Xunta de Galicia.

Becoña, E. (1993b). The prevalence of pathological gambling in Galicia (Spain). Journal of Gambling Studies, 9, 353-369.
Becoña, E. (1996). La ludopatía. Madrid: Aguilar.

Becoña, E. (1997). Pathological gambling in Spanish children and adolescents: An emerging problem. Psychological Reports, 81, 275-287.

Becoña, E. (1999). Epidemiología del juego patológico en España. Anuario de Psicología, 20, 7-19.

Becoña, E., Labrador, F.J. , Echeburúa, E. , Ochoa, E., y Vallejo, M.A. (1995). Slot gambling in Spain: A new and important social problem. Journal of Gambling Studies, 11, 265-286.

Becoña, E., Lorenzo, M.C., y Fuentes, M.J. (1996). Pathological gambling and depression. Psychological Reports, 78, 635-640.

Berg, C., y Kühlhorn, E. (1994). Social, psychological and physical consequences of pathological gambling in Sweden. Journal of Gambling Studies, 10, 275-285.

Blaszczynski, A., McConaghy, N., y Frankova, A. (1990). Boredom proneness in pathological gambling. Psychological Reports, $67,35-42$.

Brown, R.I.F. (1986). Arousal and sensation seeking components in the general explanation of gambling and gambling addiction. International Journal of Addictions, 21, 1001-1016.

Brown, R.I.F. (1987a). Classical and operant paradigms in the management of gambling addictions. Behavioural Psychotherapy, 15, 111-122.

Brown, R.I.F. (1987b). Gambling addictions, arousal and an effective decision making explanation of behavioural reversions and relapses. International Journal of Addictions, 22, 1053-1067.

Brown, R.I.F. (1988). Reversal theory and subjective experience in the explanation of addiction and relapse. En M.J. Apter, J.H. Kerr y M.P. Cowles (Eds.), Progress in reversal theory (pp. 191-211). North, Holland: Elsevier Science Publishers.

Carrasco, M.A., del Barrio, V., y Rodríguez, J.F. (2000). Sintomatología depresiva en escolares de 12 a 16 años y su relación con las distorsiones cognitivas. Revista de Psicopatología y Psicología Clínica, 5, 45-70.

Cicchetti, D., y Toth, S.L. (1998). The development of depression in children and 
adolescents. American Psychologist, 53, 221-241.

Chartier, G.M., y Larsen, M.K. (1994). Adolescent depression: CDI norms, suicidal ideation and (weak) gender effects. Adolescence, 29, 859-864.

Comisión Nacional del Juego (2000). Memoria 1999. Madrid: Ministerio de Justicia e Interior, Comisión Nacional del Juego.

Crockford, D.N., y el-Guebaly, N. (1998). Psychiatric comorbidity in pathological gambling: A critical review. Canadian Journal of Psychiatry, 43, 43-50.

Del Barrio, V. (1996). Inventario de depresión infantil, $C D I$, version experimental. Manuscrito no publicado, UNED, España.

Del Barrio, V., y Moreno, C. (1996). Evaluación de la ansiedad y depresión infantil. Ansiedad y Estrés, 2, 131-158.

Dickerson, M.G., y Adcock, S.A. (1987). Mood, arousal and cognitions in persistent gambling: Preliminary investigation of a theoretical model. Journal of Gambling Behaviors, 3, 3-15.

Fernández-Montalvo, J., y Echeburúa, E. (1997). Manual práctico del juego patológico. Madrid: Pirámide.

Fisher, S. (1999). A prevalence study of gambling and problem gambling in British adolescents. Addiction Research, 7, 509-538.

Gambino, B. (1997). Method, method: Who's got the method? What can we KNOW about the number of compulsive gamblers? Journal of Gambling Studies, 13, 291-296.

García, J.L., Díaz, C., y Aranda, J.A. (1993). Trastornos asociados al juego patológico. Anales de Psicología, 9, 83-92.

Gerstein, D., Murphy, S., Toce, M., Hoffmann, J., Palmer, A., Johnson, R. et al. (1999). Gambling impact and behavior study. Report to the National Gambling Impact Study Commission. Chicago, IL: National Opinion Research Center at the University of Illinois.

Govoni, R., Rupcich, N., y Frisch, G.R. (1996). Gambling behavior of adolescent gamblers. Journal of Gambling Studies, 12, 305-317.

Griffiths, M. (1995). Adolescent gambling. Londres: Routledge.

Gupta, R., y Deverensky, J.L. (1998). Adolescent gambling behavior: A prevalence study and examination of the correlates associated with problem gambling. Journal of Gambling Studies, 14, 319-345.

Hand, I. (1998). Pathological gambling: A negative state model and its implications for behavioral treatments. CNS Spectrums, 3, 58-71.

Hankin, B.L., Abramson, L.Y., Moffitt, T.E., Silva, P.A., McGee, R., y Angell, K.E. (1998). Development of depression from preadolescence to young adulthood: Emerging gender differences in a 10-year longitudinal study. Journal of Abnormal Psychology, 107, 128-140.

Harrington, R., Rutter, M., y Fombonne, E. (1996). Developmental pathways in depression: Multiple meanings, antecedents, and endpoints. Development and Psychopathology, 8, 601-616.

Jacobs, D.F. (1986). A general theory of addictions: A new theoretical model. Journal of Gambling Behavior, 2, 15-31.

Jacobs, D.F. (1987). A general theory of addictions: Application to treatment and rehabilitation planning for pathological gamblers. En T. Galski (Ed.), The handbook of pathological gambling (pp. 169194). Springfield, IL: Charles C. Thomas Publisher.

Jacobs, D.F. (1989). A general theory of addictions: Rationales for and evidence supporting a new approach for understanding and treating addictive behaviors. En H.J. Shaffer, S.A. Stein, B. Gambino y T.N. Cummings (Eds.), Compulsive gambling. Theory, research, and practice (pp. 35-64). Lexington, MA: Lexington Books.

Kessler, R. C., McGonagle K. A., Zhao, S., Nelson, C. B., Hughes M., Eshleman, S., Wittchen, H. U., y Kendler, K. S. (1994). Lifetime and 12-month prevalence of DSM-III-R psychiatric disorders in the United States: Results from the National Comorbidity Survey. Archives of General Psychiatry, 51, 8-19.

Kovacs, M. (1992). Children's depression inventory, CDI. Toronto, Canadá: MultiHealth Systems, Inc.

Kovacs, M., y Beck, A.T. (1977). An empirical-clinical approach toward a definition of childhood depression. En J.G. Schulterbrandt y A. Raskin (Eds.), Depression in childhood: Diagnosis, treatment and con- 
ceptual models. Nueva York, NY: Raven Press.

Kovacs, M., Freinberg, T.L., y Crouse-Noval, M.A. (1984). Depressive disorders in childhood: A longitudinal prospective study of characteristics and recovery. Archives of General Psychiatry, 41, 229-237.

Labrador, F.J., y Becoña, E. (1994). Juego patológico: aspectos epidemiológicos y teorías explicativas. En J.L. Graña (Coord.), Conductas adictivas. Teoría, evaluación y tratamiento (pp. 495-520). Madrid: Debate.

Ladouceur, R., y Mireault, C. (1988). Gambling behavior among high school students in the Quebec-area. Journal of Gambling Behavior, 4, 3-12.

Lesieur, H.R., y Blume, S.B. (1987). The South Oaks Gambling Screen (SOGS): A new instrument for the identification of pathological gamblers. American Journal of Psychiatry, 144, 1184-1188.

Lesieur, H.R., y Blume, S.B. (1993). Revising the South Oaks Gambling Screen in different settings. Journal of Gambling Studies, 9, 213-223.

Lesieur, H.R., Cross, J., Frank, M., Welch, M., White, C.M., Rubenstein, G., Moseley, K., y Mark, M. (1991). Gambling and pathological gambling among university students. Addictive Behaviors, 16, 517-527.

Lewinsohn, P.M., y Clarke, G.N. (2000). Tratamientos psicosociales para la depresión en adolescentes. Psicología Conductual, 8 , 469-485.

Méndez, X. (1998). El niño que no sonríe. Estrategias para superar la tristeza y la depresión infantil. Madrid: Pirámide.

Méndez, X, Moreno, P.J., Sánchez-Meca, J., Olivares, J., y Espada, P.J. (2000). Eficacia del tratamiento psicológico de la depresión infantil y adolescentes: una revisión cualitativa y cuantitativa de dos décadas de investigación. Psicología Conductual, 8, 487-510.

McCormick, R.A., Russo, A.M., Ramirez, L.F., y Taber, J.I. (1984). Affective disorders among pathological gamblers in treatment. American Journal of Psychiatry, 141, 215218.
National Research Council (1999). Pathological gambling: A critical review. Washington, DC: Author.

Ochoa, E., Labrador, F.J., Echeburúa, E., Becoña, E., y Vallejo, M.A. (1994). Juego patológico. Barcelona: Plaza \& Janés.

Olmedo, M., del Barrio, V. y Santed, M.A. (2000). Sexo y emoción previa como predictores del cambio en depresión y ansiedad en adolescentes. Ansiedad y Estrés, 6, 47-60.

Polaino, A., y García-Villamisar, A. (1993). La depresión infantil en Madrid. Madrid: Editorial AC.

Secades, R., y Villa, A. (1998). El juego patológico. Prevención, evaluación y tratamiento en la adolescencia. Madrid: Pirámide.

Sproston, K., Erens, B., y Orford, J. (2000). Gambling behaviour in Britain: Results from the british gambling prevalence study. Londres, RU: National Center for Social Research.

Westplal, J.R., Rush, J.A., Stevens, L., y Johnson, L.J. (2000). Gambling behavior of Louisiana students in grades 6 through 12. Psychiatric Services, 51, 96-99.

Winters, K.C. (2000, junio). Youth gambling: Transition from adolescence to young adulthood. Comunicación presentada en el 11th International Conference on Gambling \& Risk Taking.

Winters, K.C., Stinchfield, R.D., y Fulkerson, J. (1993). Toward the development of an adolescent gambling problem severity scale. Journal of Gambling Studies, 9, 63-84.

Woody, R.T.A., y Griffiths, M.D. (1998). The acquisition, development and maintenance of lottery an scratchcard gambling in adolescent. Journal of Adolescence, 21, 265-273.

Yaffee, R.A., y Brodsky, V.J. (1997). Recommendations for research and public policy in gambling studies. Journal of Gambling Studies, 13, 309-316.

Zitzow, D. (1992). Incidence and comparative study of compulsive gambling behaviors between Indian and non-Indians within and near a northern plains reservation. Bemidji, MN: Indian Health Service, Bemidji Area Office. 